\title{
Psychosocial Distress and Blood Pressure among Young Adults: Role of Cortisol as Mediator
}

Wan Fatein Nabeila W.O. ${ }^{a}$, Azarisman S.M.S. ${ }^{b}$, Jamalludin A.R. ${ }^{c}$, Norlelawati A.T. ${ }^{d}$, Ramli M. ${ }^{e}$, Aida N.S.M.S $S^{b}$, Sithu A. ${ }^{a}$, Aszrin A. ${ }^{a}$

aDepartment of Basic Medical Sciences, Kulliyyah of Medicine, International Islamic University Malaysia, Jalan Sulan Ahmad Shah, Bandar Indera Mahkota, 23200 Kuantan, Pahang, Malaysia.

bepartment of Internal Medicine, Kulliyyah of Medicine, International Islamic University Malaysia, Jalan Sulan Ahmad Shah, Bandar Indera Mahkota, 23200 Kuantan, Pahang, Malaysia.

'Department of Community Medicine, Kulliyyah of Medicine, International Islamic University Malaysia, Jalan Sulan Ahmad Shah, Bandar Indera Mahkota, 23200 Kuantan, Pahang, Malaysia.

dDepartment of Pathology and Laboratory Medicine, Kulliyyah of Medicine, International Islamic University Malaysia, Jalan Sulan Ahmad Shah, Bandar Indera Mahkota, 23200 Kuantan, Pahang, Malaysia.

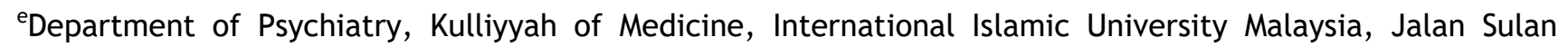
Ahmad Shah, Bandar Indera Mahkota, 23200 Kuantan, Pahang, Malaysia.

\section{ABSTRACT}

Introduction: Hypertension is the most prevalent risk factor of cardiovascular diseases in Malaysia. $17.3 \%$ of hypertension cases in Malaysia is attributed to adults aged 18 to 39 years. Psychosocial distress is a possible risk factor for elevated blood pressure in young adults, and cortisol could be the mediating factor. The aim of this study is to evaluate the mediating role of cortisol in hypertension and psychosocial distress in young adults. Methods and materials: A comparative cross-sectional study was conducted in 240 young adults aged 18 to 45 years. The body mass index, waist circumference and blood pressure parameters were recorded. Serum cortisol, creatinine, fasting blood glucose and lipid profile were measured following acute mental stress test. Psychosocial distress was assessed using the DASS-21 questionnaire. Results: Mean (standard deviation) values for SBP, DBP, MAP were 126.0(16.3), 84.1(12.2) and $98.1(13.1) \mathrm{mmHg}$ respectively. Anxiety was significantly associated with systolic blood pressure $(B=0.644)$, diastolic blood pressure $(B=0.454)$ and mean arterial pressure $(B=0.516)$ after adjusting for sex, age and cortisol. However, it was not mediated by cortisol. Depression and stress were not found to have any effect on blood pressure of the young adults studied. Conclusion: The data suggest that there is no elevated risk for psychosocial distress and hypertension that cortisol poses in young adults.

KEYWORDS: Psychosocial distress, Cortisol, Blood Pressure, Young adults, Mediation.

\section{INTRODUCTION}

Hypertension is a global epidemic affecting $40 \%$ of adults above 25 years old in 2008 , and is responsible for 45 and $51 \%$ of ischemic heart disease and stroke death, respectively. ${ }^{1}$ The number in Malaysia is

Corresponding author:

Asst. Prof. Dr. Aszrin Abdullah

Department of Basic Medical Sciences,

Kulliyyah of Medicine,

International Islamic University Malaysia,

Jalan Sulan Ahmad Shah, Bandar Indera Mahkota,

23200 Kuantan, Pahang, Malaysia.

Tel. No: +60139230011

Email: draszrin@iium.edu.my higher than the average among South East Asian countries (43 vs. $37 \%) .{ }^{1,2}$ Hence, putting it as the most prevalent cardiovascular risk factor (55\%) among Malaysians as well as among acute coronary syndrome patients $(65 \%)$, overcoming smoking, which is predominant elsewhere (62\%). ${ }^{3-5}$ Noticeably, $17.3 \%$ of hypertension cases in Malaysia is attributed to adults age 18 to 39 years. ${ }^{6}$ It is disputed that additional factors could be contributing to the high numbers of young adults with above-normal blood pressure. Being a young adult is the epitome of progression and advancement in life including career, family, financial and social status, before reaching stability in later life. During this challenging and exciting period, many would have endured increasing psychosocial distress from the extra workload, unmet 
expectation and fragmented emotion. Hence, psychosocial distress could be the perpetrator leading to elevated blood pressure.

A number of studies have looked into the likelihood of having high psychosocial distress among subjects with essential hypertension with conflicting findings. ${ }^{7-11}$ Two most commonly associated psychosocial factors were chronic stress, and nonadaptive stress response or low resilience. ${ }^{12-15}$ Specifically, among middle-age population, stress was found to be a risk factor for hypertension among females, but not males (OR 1.231-1.285). ${ }^{16}$ Nonetheless, studies looking at psychosocial factors and blood pressure among young adults are still lacking.

The sympathetic-neuroendocrine over-reactivity was hypothesised as a chief reason linking psychosocial distress to hypertension. ${ }^{13,17,18}$ Specifically, in young adults, higher salivary cortisol levels predicted higher blood pressure only in subjects with agonistic striving, but not with depressive symptoms. ${ }^{19}$ Nevertheless, it is not known if elevated cortisol further aggravates the associative effect of psychosocial stress on blood pressure. The aim of this study is to evaluate the mediating role of cortisol in hypertension and psychosocial distress in young adults. Eventually, it is hoped by identifying the link between psychosocial distress, cortisol and blood pressure, we could highlight the importance of maintaining mental well-being as a prevention of hypertension in young adults.

\section{MATERIALS AND METHODS}

This is a comparative cross-sectional study done in Kuantan, Pahang, Malaysia. Subjects were recruited by purposive sampling according to their blood pressure status as outlined by the Clinical Practice Guidelines on Management of Hypertension $4^{\text {th }}$ edition. $^{20}$ The study protocol was reviewed and approved by the Ministry of Health Malaysia Research Ethics Committee (NMRR-16-2572-32869) and International Islamic University Malaysia Research Ethics Committee (IREC 544).

\section{Subject Recruitment}

Two hundred and forty subjects aged between 18 to 45 years were recruited from several public health screening programs and primary health care clinics. Subjects with previous diagnosis of hypertension or has been on anti-hypertensive medications, history of chronic diseases, including acute coronary syndrome, diabetes mellitus, autoimmune disease, epilepsy, endocrine diseases, including Cushing's syndrome and Conn's disease, neoplasm and psychiatric comorbidity, women on hormonal contraception or pregnant, patients on corticosteroid medication, and those with evidence of secondary hypertension were excluded from the study.

\section{Study Protocol}

Subjects were given an appointment to attend the Clinical Trial Unit at International Islamic University Malaysia, Kuantan Campus between 8 to 9 am. They were fasted overnight prior to the appointment and advised to avoid strenuous work and alcohol consumption for 24 hours prior to the appointment. All participants were provided with an information sheet and written informed consent was obtained.

Social-demographic variables measured included age, sex, race, smoking status, alcohol intake status, body mass index (BMI) and waist circumference. Blood pressure was measured using recently validated automated digital blood pressure monitor (Omron HEM-7130) and average blood pressure was taken on three different occasions (screening, sample collection and blood results consultation day.)

Mean arterial pressure (MAP) was derived from the formula, MAP $=\square$ Systolic blood pressure + Diastolic blood pressure $(\mathrm{mmHg})$. Stroop Colour and Word Test (SCWT) were conducted prior to blood-taking to induce acute mental stress. Ten millilitres of venous blood were collected for biochemical tests, including serum creatinine, fasting blood glucose, fasting lipid profile, and serum cortisol. Psychosocial distress was measured by selfadministered, validated Bahasa Melayu version DASS -21 Questionnaire. ${ }^{21}$ DASS-21 Questionnaire is a 4-point severity scale of 21 questions measuring depression, anxiety and stress over the past one week, and were comparable to the 42 item questionnaire. $^{21}$ 


\section{Statistics}

The data was analysed using IBM SPSS Statistics 22.0 (IBM Corp, Armonk, New York). All numerical data were described using mean (standard deviation). The associations among psychosocial distress, cortisol and blood pressure parameters were evaluated using general linear model. Subsequently, the mediation analysis of the role of cortisol in the associations between psychosocial distress and blood pressure parameters, were tested using PROCESS Macro version 2.16 for SPSS by Andrew F. Hayes. ${ }^{22}$ All models were adjusted for age, sex and fasting blood glucose. The theoretical model is shown in Fig. 1. $p<0.05$ was considered statistically significant.

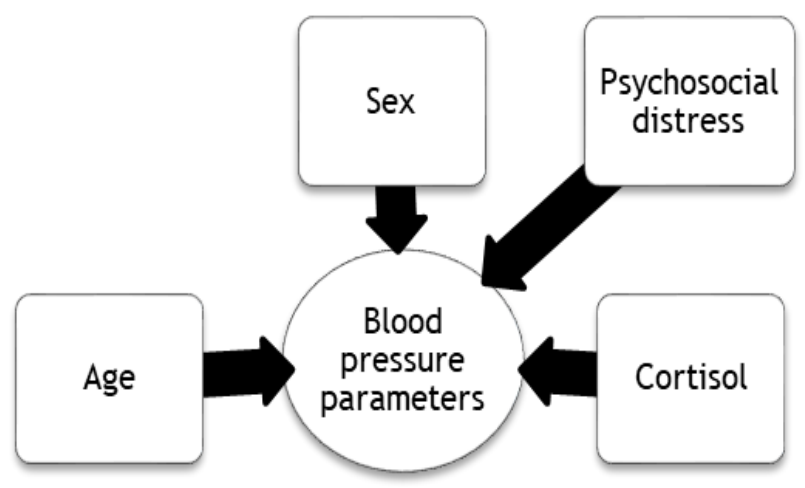

Figure 1: Theoretical model of the factors affecting blood pressure parameters. The factors tested include age, sex, psychosocial distress and serum cortisol. This model also includes the hypothesized mediating role of cortisol on the association of psychosocial distress with blood pressure parameters. Psychosocial distress components were depression (D), anxiety (A) and stress (S) as measured by DASS-21 questionnaire. Blood pressure parameters include systolic blood pressure (SBP), diastolic blood pressure (DBP) and mean arterial pressure (MAP).

\section{RESULTS}

The subjects were distributed between 18 to 45 years old with the mean age of 32.4(6.9) years, $63.8 \%$ were male and $91.3 \%$ were Malay (Table I). Mean (standard deviation) values for SBP, DBP and MAP were 126.0(16.3), 84.1(12.2) and 98.1(13.1) $\mathrm{mmHg}$ respectively.

\section{Associations between psychosocial distress and blood pressure parameters}

The anxiety level was positively associated with SBP $(B=0.644, p=0.032)$, DBP $(B=0.230, p=0.049)$ and MAP $(B=0.516, p=0.035)$. No significant association between depression and stress level, with any blood pressure parameters were found after adjusting for age and sex. After adjusting for sex, age and fasting blood glucose, the cortisol level was not associated with SBP, DBP and MAP (Table II).

Table I: Sociodemographic distribution of the subjects

\begin{tabular}{|c|c|}
\hline Sociodemographic aspect & $\mathrm{n}(\%)$ \\
\hline Age (years) & $32.5(6.9)^{a}$ \\
\hline Male & $153(63.8)$ \\
\hline \multirow[t]{2}{*}{ Race } & $220(91.7)$ \\
\hline & $20(8.3)$ \\
\hline Smoking & $54(22.5)$ \\
\hline Alcohol intake & $10(4.2)$ \\
\hline Body mass index $\left(\mathrm{kg} / \mathrm{m}^{2}\right)$ & $27.5(5.7)^{\mathrm{a}}$ \\
\hline Waist circumference $(\mathrm{cm})$ & $90.5(12.9)^{\mathrm{a}}$ \\
\hline Female & $86.1(11.1)^{\mathrm{a}}$ \\
\hline Male & $93.1(13.2)^{\mathrm{a}}$ \\
\hline
\end{tabular}

${ }^{a}$ Mean (Standard deviation).

Mediation analysis of the role of cortisol in the association between psychosocial distress and blood pressure parameters

The mediation model for the effect of cortisol for an association between depression/anxiety/stress and blood pressure parameters is shown in Figure 2 with standardised regression coefficients. There was no significant association between depression (Figure 2a $-c$ ), anxiety (Figure 2d-f) and stress (2g-i), with serum cortisol level, after adjusting for sex, age and fasting blood glucose. After adjusting for age, sex, fasting blood glucose and each component of psychosocial distress, we found that cortisol was not significantly associated with SBP, DBP or MAP (Figure $2)$, however the trend appears to be approaching significance ( $p$ ranges from 0.052 to 0.076 ).

The direct effect of depression and stress, on SBP, DBP and MAP were not significant (Figure $2 \mathrm{a}-\mathrm{c}, 2 \mathrm{~g}-\mathrm{i}$ ). However, anxiety had a significant direct effect on MAP $(B=0.500, p=0.043)$, while the direct effect on SBP and DBP were approaching significance $(p=0.051)$ (Figure $2 d-f)$. On the other hand, indirect 
effect output indicated that there was no mediation effect of cortisol in the association between anxiety and MAP (indirect effect $=-0.0012,95 \% \mathrm{Cl}=[-0.091$, $0.071])$. Overall, serum cortisol did not mediate the association between psychosocial distress and blood pressure parameters.

Table II: $p$-value of association between psychosocial distress and cortisol level with blood pressure, and psychosocial distress to cortisol level using general linear model.

\begin{tabular}{llll} 
& \multicolumn{1}{l}{$\begin{array}{l}\text { p-value (B) after adjusting for age and } \\
\text { sex }\end{array}$} & & \\
\cline { 2 - 3 } & $\begin{array}{l}\text { Systolic } \\
\text { blood pres- } \\
\text { sure (SBP) }\end{array}$ & $\begin{array}{l}\text { Diastolic } \\
\text { blood pres- } \\
\text { sure (DBP) }\end{array}$ & $\begin{array}{l}\text { Mean } \\
\text { arterial } \\
\text { pressure } \\
\text { (MAP) }\end{array}$ \\
Depression & 0.307 & 0.298 & 0.285 \\
Anxiety level & $0.032^{*}$ & $0.049^{*}$ & $0.035^{*}$ \\
& $(0.644)$ & $(0.230)$ & $(0.516)$ \\
Stress level & 0.353 & 0.141 & 0.193 \\
Cortisol level & $0.156^{\theta}$ & $0.118^{\theta}$ & $0.118^{\theta}$ \\
\hline
\end{tabular}

*(B) was mentioned when $p<0.05 .{ }^{\theta}$ Adjusted for age and sex.

\section{DISCUSSION}

In this study, our analysis showed that anxiety was positively associated with SBP, DBP and MAP whereas depression and stress were not. Moreover, the data suggest that anxiety had a significant direct effect on MAP only, and cortisol had no mediating role in the association. The significant relationship between anxiety and blood pressure is consistent with the findings of previous studies in a middle-aged Dutch cohort (mean age 58.2 years) and among European students (mean age 23.9 years)..$^{23,24}$ This positive association highlights the importance of managing anxiety among young adults. Nevertheless, one study had previously reported an inverse relationship between anxiety and blood pressure among migrants in Ghanaian with high prevalence of hypertension $(54 \%) .{ }^{10}$ With regards to stress, our finding was in contrast to many studies which reported that stress is a risk factor for hypertension. ${ }^{12,14,16,24,25}$ The nonsignificant mediation by cortisol in the association between psychological distress and blood pressure parameters suggest that changes in serum cortisol in distressed young adults do not further affect the blood pressure.
The difference in results could be due to the different psychological tool used to measure psychological stress, and the duration of experiencing distress prior to the study. Among other tools used by other groups were Composite International Diagnostic Interview Short-Form for Major Depression, The Anxiety Scale of the Italian Version of the General Health Questionnaire (GHQ12) and 6-item Kessler Psychological Distress Scale. The current study utilised DASS-21 questionnaire which measured the depression, anxiety and stress over the past week. Furthermore, our hypertensive samples were newly-diagnosed who have yet to receive anti-hypertensive treatment.

Based on our finding, a recent psychological distress does not cause a sudden increase in blood pressure. While our findings were in contrast to the findings of other researchers that reported positive association between chronic psychological distress and blood pressure, we suggest that the association could be reciprocal, i.e., in a long run, the psychological distress could cause an increase in blood pressure. Due to the cross-sectional study design, we cannot rule out the causal link and alternative mechanisms.

In the present study, cortisol was not associated with any of the blood pressure parameters when adjusted to each psychosocial distress although the trends were approaching significance. This is in contrast to the hypothesis that cortisol reactivity could modulate blood pressure. ${ }^{26}$ We postulate that this finding could potentially change if the study was carried out in a larger sample size. The nonsignificant mediation by cortisol in the association between psychological distress and blood pressure parameters suggest that changes in serum cortisol in distressed young adults do not further affect the blood pressure. Finally, it would also be beneficial if we could assess serum cortisol variability and quantitate the different sites of cortisol such as the urinary or salivary cortisol.

This study attended current gaps in the literature in a few ways. We have narrowed down our samples to young adults which overcome the lack of literature on studies of essential hypertension in young adults, and we only included newly-diagnosed hypertensive samples which excluded the compounding effect of anti-hypertensive medication intake and hypertension-related complications from the analysis. 


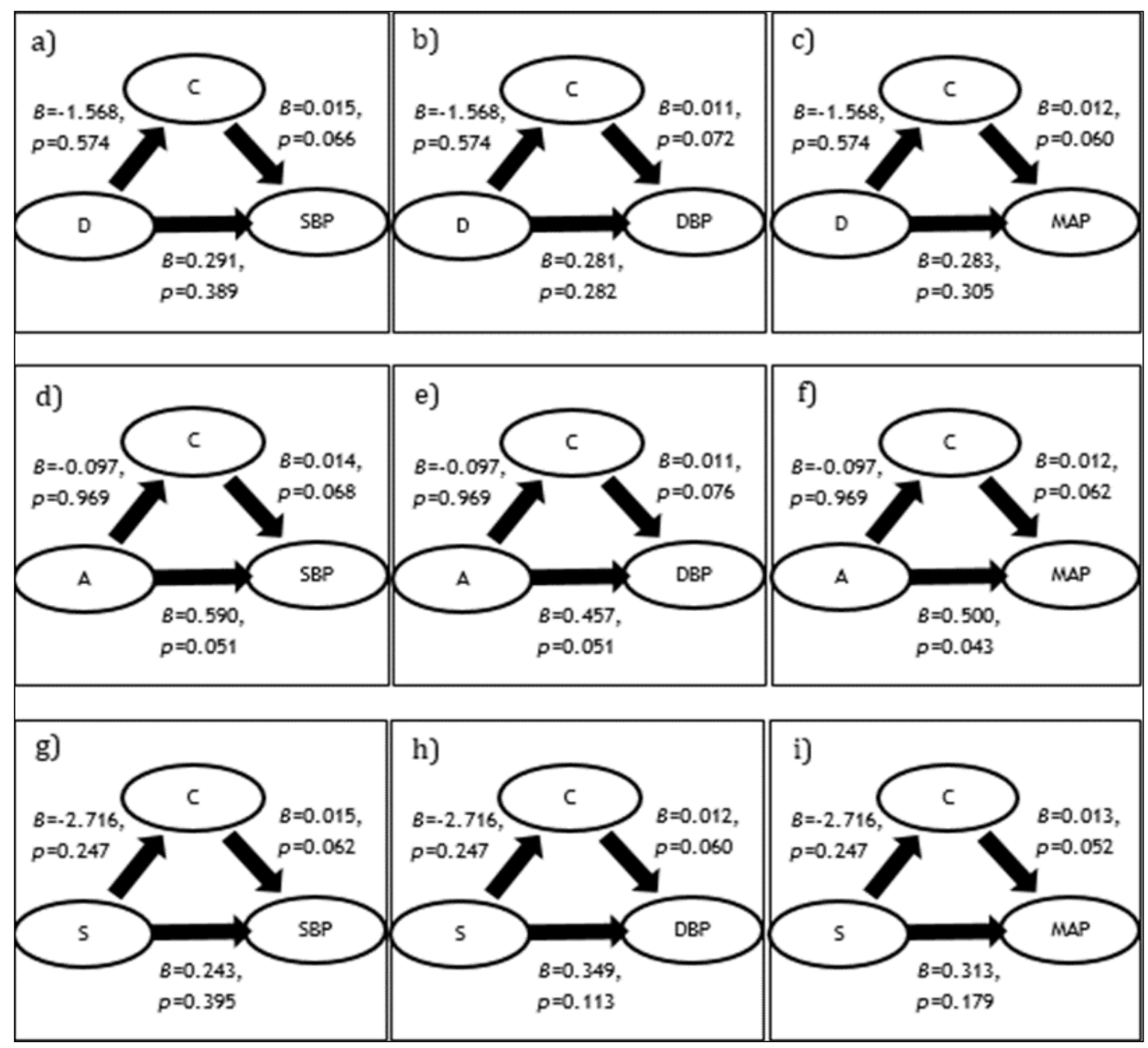

Figure 2: Results of the mediation model for each psychosocial distress component. The path weights in the graphs were standardised. (a)(b)(c) represents the mediation model of cortisol in the association between depression and blood pressure parameter, $(\mathrm{d})(\mathrm{e})(\mathrm{f})$ represents the mediation model of cortisol in the association between anxiety and blood pressure parameters, $(\mathrm{g})(\mathrm{h})(\mathrm{i})$ represents the mediation model of cortisol in the association between stress and blood pressure parameters. $\mathrm{D}=$ Depression. $\mathrm{A}=$ Anxiety. $\mathrm{S}=$ Stress. $\mathrm{C}=$ Serum cortisol. $\mathrm{SBP}=$ Systolic blood pressure. $\mathrm{DBP}=$ Diastolic blood pressure. MAP $=$ Mean arterial pressure. $B=$ standardised coefficient. All models are adjusted for age, sex and fasting blood glucose.

Also, we address the question of the interrelations between psychosocial distress, cortisol and blood pressure which has not been studied elsewhere. Additionally, we measured SBP, DBP and MAP as blood pressure parameters to allow an inclusive review of the effect of psychosocial distress on blood pressure. Furthermore, many groups focused on one component of psychosocial distress, while we measured multiple components; depression, anxiety and stress, which enabled us to independently evaluate the directions of the association of each component.

\section{LIMITATIONS OF THE STUDY}

One of the limitations of this study is that the forte of the findings was restricted by the crosssectional observational study design.

Second, the current study's sample size is limited to $n=240$, and we cannot ensure that the direction would remain when the effect was studied in a larger sample size. Longitudinal study using a larger sample size is obliged to further describe the direction and magnitude of effects, and to satisfy the generalizability of findings. Third, the psychosocial distress described in this study was only of the recent one week. Hence, we cannot rule out the possibility that an acute or long-term distress could be differently associated with cortisol and blood pressure. Therefore, the proposed mediational pathway are subject to be tested with different 
intervals of psychosocial distress. Finally, we could not exclude the white-coat effect during which the blood pressure was measured, although we have tried to minimize the effect by taking three measurements at different instances.

Despite the limitations, the current study contributes to the literature by refining the associations between psychosocial distress, cortisol and blood pressure in young adults. Our findings suggest that in managing young adults with high blood pressure, emphasis should be made on the psychological aspect of their management, rather than relying merely on the blood results and pharmacotherapy.

\section{CONCLUSION}

The current study showed that there is no elevated risk for psychosocial distress and hypertension that cortisol poses in young adults.

\section{CONFLICT OF INTEREST}

The authors declared no conflict of interest.

\section{ACKNOWLEDGEMENTS}

This study was jointly funded by the Ministry of Higher Education Malaysia (FRGS16-056-0555) an International Islamic University Malaysia (RIGS15076-0076, RIGS15-077-0077)

\section{REFERENCES}

1. World Health Organisation. A Global Brief on Hypertension [online]. Available at: http:// ish-world.com/downloads/pdf/ global_brief_hypertension.pdf. Accessed March 17, 2017.

2. Naing C, Yeoh PN, Wai VN, et al. Hypertension in Malaysia. Medicine 2016; 95:e2417.

3. Selvarajah S, Kaur G, Haniff J, et al. Comparison of the Framingham Risk Score, SCORE and WHO/ISH cardiovascular risk prediction models in an Asian population. Int J Cardio 2014; 176:211-8.

4. Wan Ahmad WA, Sim KH, eds. Annual Report of The NCVD-ACS Registry, 2011-2013. Kuala Lumpur, Malaysia: National Cardiovascular Disease Database, 2015.

5. Fox K, Eagle K, Gore J, et al. The Global Registry of Acute Coronary Events, 1999 to
2009 - GRACE. Heart 2010; 96:1095-101.

6. Azahadi Omar M, Irfan NI, Yi KY, et al. Prevalence of Young Adult Hypertension in Malaysia and Its Associated Factors: Findings From National Health and Morbidity Survey 2011. MJPHM 2016; 16:274-83.

7. Spruill TM. Chronic psychosocial stress and hypertension. Curr Hypertens Rep 2010; 12:10-6.

8. Kessler RC, Ormel J, Demler O, Stang PE. Comorbid Mental Disorders Account for the Role Impairment of Commonly Occurring Chronic Physical Disorders: Results from the National Comorbidity Survey. J Occup Environ Med 2003; 45:1257-66.

9. Carroll D, Phillips AC, Gale CR, et al. Generalized Anxiety and Major Depressive Disorders, Their Comorbidity and Hypertension in Middle-Aged Men. Psychosom Med 2010; 72: 16-9.

10. Agyei B, Nicolaou $M$, Boateng $L$, et al. Relationship between psychosocial stress and hypertension among Ghanaians in Amsterdam, the Netherlands - The GHAIA study. BMC Public Health 2014;14:1.

11. Bhat SK, Beilin LJ, Robinson M, et al. Relationships between depression and anxiety symptoms scores and blood pressure in young adults. J Hypertens 2017; 35:198391.

12. Sparrenberger F, Cichelero FT, Ascoli AM, Fonseca FP, Weiss G, Berwanger O, et al. Does psychosocial stress cause hypertension? A systematic review of observational studies. J Hum Hypertens 2009; 23:12-9.

13. Sztejfman C. Psychosocial Stress and Low Resilience : A Risk Factor for Hypertension . Relations between Hypertension and Psychoanalysis. Rev Argent Cardiol 2010; 78: editorial.

14. Liu MY, Li N, Li WA, Khan H. Association between psychosocial stress and hypertension: a systematic review and metaanalysis. Neurol Res 2017; 39:573-80.

15. Cuffee $Y$, Ogedegbe C, Schoenthaler A. Psychosocial Risk Factors for Hypertension : an Update of the Literature. Curr Hypertens Rep 2014; 16:1-11.

16. Hu B, Liu X, Yin S, et al. Effects of psychological stress on hypertension in middle-aged Chinese: A cross-sectional study. PLOS ONE 2015; 10:1-13. 
17. Wirtz PH, Ehlert U, Bärtschi C, et al. Changes in plasma lipids with psychosocial stress are related to hypertension status and the norepinephrine stress response. Metabolism 2009; 58:30-7.

18. Brown ES, Varghese FP, MCEwen BS. Association of depression with medical illness: does cortisol play a role? Biol Psychiatry 2004; 55:1-9.

19. Ewart CK, Elder GJ, Jorgensen RS, et al. The Role of Agonistic Striving in the Association Between Cortisol and High Blood Pressure. Psychosom Med 2017; 79:416-25.

20. Ministry of Health. CPG Management of Hypertension (4th Edition). Malaysia: Ministry of Health, 2013.

21. Ramli M, Rosnani S, Fasrul AA.

Psychometric Profile of Malaysian version of the Depressive , Anxiety and Stress Scale 42-item ( DASS-42 ). MJP Online Early 2012;1:7.

22. Hayes AF.. PROCESS: A versatile computational tool for observed variable mediation, moderation, and conditional process modeling. [White paper] 2012:139. Available at: http:// www.afhayes.com/ public/ process2012.pdf. Accessed July 28, 2018.

23 Ginty AT, Carroll D, Roseboom TJ, et al. Depression and anxiety are associated with a diagnosis of hypertension 5 years later in a cohort of late middle-aged men and women. J Hum Hypertens 2013; 27:187-90.

24. Mucci N, Giorgi G, Ceratti SDP, et al. Anxiety, stress-related factors, and blood pressure in young adults. Front Psychol 2016; 7:1-10.

25. Murray E, Nina E, Markus S, et al. Chronic Mental Stress Is A Cause Of Essential Hypertension: Presence Of Biological Markers Of Stress. Clin Exp Pharmacol Physiol 2008; 35:498-502.

26. Hamer M, Steptoe A. Cortisol responses to mental stress and incident hypertension in healthy men and women. J Clin Endocrinol Metab 2012; 97:29-34. 
